\title{
Prescribing and borderline personality disorder
}

\section{SUMMARY}

Accurate diagnosis is fundamental to effective management of borderline personality disorder, but many patients remain undetected.

The first-line management for borderline personality disorder is psychosocial treatment, not drugs. There are major prescribing hazards including polypharmacy, overdose and misuse.

Drug treatment might be warranted for patients who have a co-occurring mental disorder such as major depression.

If a drug is prescribed for borderline personality disorder, it should only be as an adjunct to psychosocial treatment. There should be clear and collaborative goals that are regularly reviewed with the patient.

Use single drugs prescribed in limited quantities for a limited time. Stop drugs that are ineffective.

\section{Introduction}

Borderline personality disorder is a severe mental disorder that has its onset during adolescence and emerging adulthood.' It affects up to $3 \%$ of the population ${ }^{2,3}$ and occurs almost equally among males and females. ${ }^{4}$

The disorder has a fourfold higher prevalence among primary care patients than among the general population. ${ }^{5}$ It affects around one-quarter of primary care patients with depression ${ }^{6}$ and one in five psychiatric outpatients. ${ }^{7,8}$ In these settings females outnumber males by a ratio of up to $4: 1$.

Borderline personality disorder is a leading contributor to the burden of disease in our community. It is associated with adverse longterm outcomes that include severe and persistent functional disability, ${ }^{9}$ high family and carer burden, ${ }^{10}$ physical ill health, ${ }^{11}$ and premature mortality, ${ }^{12}$ including a suicide rate of $8 \% .^{13}$ People with the disorder use mental health services continuously for long periods of time. ${ }^{14}$ After schizophrenia, borderline personality disorder is the most costly psychiatric disorder to treat in Australia on a per case basis. ${ }^{15}$

Psychosocial treatment is the primary therapy but access to this is poor. Despite the effectiveness of treatment, persisting psychopathology (e.g. borderline personality disorder features, depressive and anxiety symptoms) and functional impairment remain clinically problematic.
While it is easier to provide prescriptions than psychosocial treatments, evidence does not support the use of drugs as first-line or sole treatment. ${ }^{16}$ Nevertheless, psychotropic drug use is common and needs careful management and review.

\section{Clinical presentation}

Borderline personality disorder is characterised by a pervasive pattern of instability in emotional regulation, interpersonal relationships and self-image, along with marked impulsivity. ${ }^{17}$ Clinically, this often manifests as recurrent self-harm and suicide attempts. The person frequently describes a chaotic lifestyle and relationships, reckless behaviours likely to harm the individual (e.g. impulsive substance use, unsafe sex), chronic dysphoria and anxiety, severe mood instability and reactive aggression.

People with borderline personality disorder typically present to health services during times of crisis, following self-harm, because of the consequences of impulsive and self-damaging behaviour, or because of poor physical, sexual and reproductive health. Interpersonal problems are at the very heart of borderline personality disorder and these are usually magnified during crises. For example an individual might have unrealistically high expectations or demands of care from health practitioners and when these are not met, he or she might respond aggressively. It is important to remember that clinicians are likely to see patients at their lowest ebb, but this is when they are in greatest need of timely and coherent assistance.

\section{Andrew M Chanen}

Deputy research director and Head Personality Disorder Research' Professor ${ }^{2}$

Director of Clinical Services 3

\section{Katherine $\mathbf{N}$ Thompson} Senior research fellow ${ }^{1,2}$

'Orygen - The National Centre of Excellence in Youth Mental Health

${ }^{2}$ Centre for Youth Mental Health

University of Melbourne ${ }^{3}$ Orygen Youth Health Northwestern Mental Health Melbourne

\section{Key words}

antidepressant, antipsychotic agent, borderline personality disorder, polypharmacy, psychosocia intervention strategy

Aust Prescr 2016;39:49-53 http://dx.doi.org/10.18773/ austprescr.2016.019 


\section{Assessment}

The DSM- 5 criteria for borderline personality disorder are listed in Box $1 . .^{18}$ Although the DSM-5 requires any five of these nine criteria in order to make a diagnosis, even low levels of borderline pathology (e.g. one criterion) are associated with substantial increases in psychosocial impairment. ${ }^{19}$

Despite the evidence of the reliability and validity of the diagnosis, and the treatability of the condition, ${ }^{20}$ many people with borderline personality disorder remain undiagnosed in clinical practice. This places them at risk of being given treatments that are ineffective or even harmful. ${ }^{21}$ The central task for diagnosing personality disorder is to separate 'state' (transient aberrations in mental state) from 'trait' (long-standing patterns of thinking, feeling, behaving, perceiving and relating). Many mental state disorders can present with features that are similar to borderline personality disorder. For example, affective dysregulation is characteristic of both bipolar disorder and borderline personality disorder. Also, the current definition of depression incorporates non-specific forms of dysphoria that overlap with borderline personality disorder. What distinguishes borderline personality from these other disorders is that the features are present most of the time and comprise part of the patient's 'usual self'. These patients will tell you that this is how they 'usually are'. Although various tools are available to aid the diagnosis of borderline personality disorder, clinical application of the DSM-5 criteria is sufficient in a busy clinical practice. Each of the nine criteria should be enquired about and considered in turn.

\section{Box 1 Diagnostic criteria for borderline personality disorder 18}

A pervasive pattern of instability of interpersonal relationships, self-image, and affects, and marked impulsivity, beginning by early adulthood and present in a variety of contexts, as indicated by five (or more) of the following:

1. Frantic efforts to avoid real or imagined abandonment (do not include suicidal or selfmutilating behaviour covered in criterion 5)

2. A pattern of unstable and intense interpersonal relationships characterised by alternating between extremes of idealisation and devaluation

3. Identity disturbance: markedly and persistently unstable self-image or sense of self

4. Impulsivity in at least two areas that are potentially self-damaging (e.g. spending, sex, substance abuse, reckless driving, binge eating - do not include suicidal or self-mutilating behaviour covered in criterion 5)

5. Recurrent suicidal behaviour, gestures, or threats, or self-mutilating behaviour

6. Affective instability due to a marked reactivity of mood (e.g. intense episodic dysphoria, irritability, or anxiety usually lasting a few hours and only rarely more than a few days)

7. Chronic feelings of emptiness

8. Inappropriate, intense anger or difficulty controlling anger (e.g. frequent displays of temper, constant anger, recurrent physical fights)

9. Transient, stress-related paranoid ideation or severe dissociative symptoms
A common cause of misdiagnosis of borderline personality disorder is to rely on 'gut feeling' when a patient presents as interpersonally abrasive, sullen or hostile, particularly if the individual also engages in self-harm. Such diagnoses are often unreliable because they do not assess each of the DSM-5 borderline personality disorder criteria and do not take into account other reasons for such presentations, such as temporary aberrations in mental state, depression or other disorders.

Another cause of diagnostic confusion is the high rate of comorbid conditions. Comorbidity with other personality disorders and with mental state disorders is the norm. At times, these other disorders (e.g. mood, anxiety, eating and substance use disorders) can overwhelm the clinical picture, but this does not indicate that the underlying personality pathology is unimportant or should be a secondary concern. ${ }^{21}$ Rather, there is evidence to suggest that personality disorder might be a key vulnerability factor for recurrent mental state disorders. ${ }^{22,23}$ Patients with borderline personality disorder who have these co-occurring conditions should be treated for these conditions in accordance with best practice. However, there should not be a disproportionate emphasis given to the immediate relief of mental state pathology at the expense of managing the borderline personality disorder.

\section{Treatment}

Since the 1990s there has been growing optimism and enthusiasm for the treatment of borderline personality disorder. There is now a variety of effective evidencebased psychosocial treatments (Box 2). Referral for one of these first-line treatments is recommended. ${ }^{16,24}$ No single treatment is recommended over another and they have common core features (Box 2). ${ }^{25}$ Treatment guidelines include one published by the Australian National Health and Medical Research Council (NHMRC). ${ }^{16}$

Despite advances in psychosocial treatment for borderline personality disorder, improvements remain suboptimal. ${ }^{24,25}$ Access to treatment is limited, and dropout rates are high (15-77\%). ${ }^{26}$ The NHMRC guidelines provide advice that can be implemented when referral to specialist services for borderline personality disorder is unavailable.

\section{Evidence for drug therapy}

Pharmacotherapy has been investigated as a standalone or adjunctive treatment option for borderline personality disorder. There are many high-quality reviews ${ }^{16,27,28}$ including a Cochrane review. ${ }^{27,29}$ The Cochrane group found 33 randomised controlled trials in adults with borderline personality disorder, ${ }^{27,29}$ 
but commented that the overall evidence base for prescribing is unsatisfactory. The literature is hampered by small trials (less than 50 patients) of numerous drugs, short treatment periods (mean duration of 12 weeks), diverse outcome measures, infrequent replication of findings, and lack of independence from the pharmaceutical industry. ${ }^{30,31}$ In addition, controversy has surrounded the methods of one research group and the integrity of the findings. ${ }^{29}$

The available evidence ${ }^{16,27-29}$ does not support a prominent role for selective serotonin reuptake inhibitors in the treatment of borderline personality disorder, despite their widespread use. Other drugs require further investigation. Mood stabilisers (topiramate, sodium valproate, lamotrigine) have shown some effect in reducing affective dysregulation and impulsive aggression. Antipsychotics such as aripiprazole, olanzapine and quetiapine have shown some effect in reducing cognitive-perceptual symptoms and affective dysregulation. Omega-3 polyunsaturated fatty acids might reduce the overall severity of borderline personality disorder. ${ }^{27}$

Although the evidence for drug therapy is less conclusive than for the psychosocial interventions, US data indicate that prescribing rates for borderline personality disorder are paradoxically high. Drugs are prescribed for $78 \%$ of patients for more than $75 \%$ of

\section{Box 2 Psychosocial treatments and their common characteristics}

\section{Treatments \\ Dialectical behaviour therapy \\ Mentalisation-based treatment \\ Schema-focused therapy \\ Cognitive behavioural therapy \\ Cognitive analytic therapy \\ Transference-focused therapy}

Systems training for emotional predictability and problem solving

Dynamic deconstructive psychotherapy

\section{Common characteristics ${ }^{25}$}

Approaches to prototypic borderline personality disorder problems are structured (manual-directed)

Patients are encouraged to adopt self-control (i.e. sense of agency)

Therapists help patients to connect feelings to events and actions

Therapists are active, responsive and validating

Therapists discuss cases, including personal reactions, with others the time over a six-year period and polypharmacy occurs in $37 \%$ of patients, ${ }^{32}$ perhaps reflecting clinical needs and pressures. To date, there has been no study of Australian prescribing practices, but clinical experience suggests that the situation might be similar.

\section{Guidelines}

In light of the clinical needs and pressures, and the harm associated with prescribing for these patients, the NHMRC ${ }^{16}$ and UK National Institute for Health and Care Excellence (NICE) ${ }^{28}$ have considered the controlled trial evidence. In general, both groups recommend against prescribing psychotropic drugs for treating borderline personality disorder. Importantly, the NHMRC guideline recognises that the absence of evidence can lead to heterogeneity of practice. It therefore provides some guidance for those who wish to try empirical treatment (Box 3).

\section{Box 3 Key recommendations for treating borderline personality disorder ${ }^{16}$}

- People with borderline personality disorder should be provided with structured psychological therapies that are specifically designed for borderline personality disorder, and conducted by one or more adequately trained and supervised health professionals.

- Drugs should not be used as primary therapy for borderline personality disorder, because they have only modest and inconsistent effects, and do not change the nature and course of the disorder.

- The time-limited use of drugs can be considered as an adjunct to psychological therapy, to manage specific symptoms.

- Caution should be used if prescribing drugs that may be lethal in overdose, because of high suicide risk with prescribed drugs in people with borderline personality disorder.

- Caution should be used if prescribing medicines associated with substance dependence.

- Before starting time-limited pharmacotherapy for people with borderline personality disorder:

- ensure that a drug is not used in place of other more appropriate interventions

- take account of the psychological meaning of prescribing (both for the individual and for the prescriber) and the impact that prescribing decisions may have on the therapeutic relationship and the overall borderline personality disorder management plan, including long-term treatment strategies

- use a single drug and avoid polypharmacy if possible

- ensure that there is consensus among prescribers about the drug used, and collaboration with other health professionals involved in the person's care, and that the main prescriber is identified

- establish likely risks of prescribing, including interactions with alcohol and other substances.

- The use of drugs can be considered in acute crisis situations where psychological approaches are not sufficient.

- If drugs have been prescribed to manage a crisis, they should be withdrawn once the crisis is resolved. 


\section{The doctor-patient relationship}

All prescribing occurs in the context of the doctorpatient relationship. However, this takes on particular significance when the condition being treated is fundamentally a relational disorder. Interpersonal difficulties and challenges, particularly maladaptive help-seeking, are part of the everyday work of caring for people with borderline personality disorder. This need not be a deterrent to becoming involved in their care, but it does require a degree of self-reflection and self-monitoring.

In caring for patients with borderline personality disorder, strong thoughts or feelings on the part of the doctor can be used as a reminder to reflect on what is happening in the doctor-patient relationship: Am I feeling 'pulled' or 'pushed' to respond in particular ways? Why won't the patient do what they need to? Who is responsible for change? Am I doing all the work? Am I responding as I might usually do, or am I treating this patient differently? Some patients will invite clinicians to behave differently with them and we can be aware of these invitations without automatically feeling 'cheated' or 'manipulated' and becoming more rigid and inflexible.

\section{SELF-TEST QUESTIONS}

True or false?

5. Selective serotonin reuptake inhibitors are the first-line treatment for borderline personality disorder. 6. Binge eating may be a feature of borderline personality disorder.

Answers on page 66
Some strategies to assist in this task can be drawn from the common characteristics of evidencebased treatments in Box 2, e.g. encouraging patients to develop a sense of self-control, and helping patients to connect feelings to events and actions. Collaborative goal setting is fundamental to successful prescribing. This involves being clear about what it is that the patient is seeking, and agreeing to common goals. A key role for the clinician is to keep one's eye on these goals, assisting the patient to persist with the chosen strategy, without becoming too rigid.
Clinicians need to be aware of their own limits and needs and should be willing to communicate these to the patient, rather than dress them up as the patient's needs. For example, the doctor's responsibility to prescribe safely, legally and ethically might be at odds with the patient's demands for immediate relief from distressing symptoms.

Clinicians also need to admit and be willing to openly discuss difficulties. It is often much easier to start prescribing than to stop it. Honest and open discussion about whether a trial of a drug is warranted or has been effective is fundamental.

\section{Conclusion}

Borderline personality disorder is a leading cause of disability and mortality and is common in clinical practice. Although it was once considered 'untreatable', the outlook for patients with borderline personality disorder is much improved, with a range of effective psychosocial treatments available for the disorder. However, access to these treatments is limited in the Australian healthcare system. Despite this, drugs should not be used as primary therapy for borderline personality disorder because they have only modest and inconsistent effects. They do not change the nature or course of the disorder. A time-limited trial of a drug can be considered as an adjunct to psychological therapy, to manage specific symptoms, but caution is needed. $<$

Conflict of interest: Andrew Chanen was a member of the development group for the National Health and Medical Research Council's Clinical Practice Guideline for the Management of Borderline Personality Disorder.

Acknowledgement: The authors would like to thank Dr Louise McCutcheon for comments on an earlier version of this manuscript.

\section{REFERENCES}

1. Chanen AM, McCutcheon L. Prevention and early intervention for borderline personality disorder: current status and recent evidence. Br J Psychiatry Supp 2013;202:s24-9. http://dx.doi.org/10.1192/bjp.bp.112.119180

2. Lenzenweger MF. Epidemiology of personality disorders [vi.]. Psychiatr Clin North Am 2008:31:395-403. http://dx.doi.org/10.1016/j.psc.2008.03.003

3. Trull TJ, Jahng S, Tomko RL, Wood PK, Sher KJ. Revised NESARC personality disorder diagnoses: gender, prevalence, and comorbidity with substance dependence disorders. J Pers Disord 2010;24:412-26. http://dx.doi.org/10.1521/ pedi.2010.24.4.412

4. Torgersen S, Kringlen E, Cramer V. The prevalence of personality disorders in a community sample. Arch Gen Psychiatry 2001;58:590-6. http://dx.doi.org/ 10.1001/archpsyc.58.6.590

5. Gross R, Olfson M, Gameroff M, Shea S, Feder A, Fuentes M, et al. Borderline personality disorder in primary care. Arch Intern Med 2002;162:53-60. http://dx.doi.org/10.1001/archinte.162.1.53

6. Riihimäki K, Vuorilehto M, Isometsä E. Borderline personality disorder among primary care depressive patients: a five-year study. J Affect Disord 2014;155:303-6. http://dx.doi.org/10.1016/j.jad.2013.10.050

7. Zimmerman M, Chelminski I, Young D. The frequency of personality disorders in psychiatric patients [vi.]. Psychiatr Clin North Am 2008;31:405-20. http://dx.doi.org/10.1016/j.psc.2008.03.015
8. Chanen AM, Jovev M, Djaja D, McDougall E, Yuen HP, Rawlings D, et al. Screening for borderline personality disorder in outpatient youth. J Pers Disord 2008;22:353-64. http://dx.doi.org/10.1521/pedi.2008.22.4.353

9. Gunderson JG, Stout RL, McGlashan TH, Shea MT, Morey LC, Grilo CM, et al. Ten-year course of borderline personality disorder: psychopathology and function from the Collaborative Longitudinal Personality Disorders study. Arch Gen Psychiatry 2011;68:827-37. http://dx.doi.org/10.1001/ archgenpsychiatry.2011.37

10. Hoffman PD, Buteau E, Hooley JM, Fruzzetti AE, Bruce ML. Family members' knowledge about borderline personality disorder: correspondence with their levels of depression, burden, distress, and expressed emotion. Fam Process 2003;42:469-78. http://dx.doi.org/10.1111/j.1545-5300.2003.00469.x

11. El-Gabalawy R, Katz LY, Sareen J. Comorbidity and associated severity of borderline personality disorder and physical health conditions in a nationally representative sample. Psychosom Med 2010;72:641-7. http://dx.doi.org/ 10.1097/PSY.0b013e3181e10c7b

12. Fok ML, Hayes RD, Chang CK, Stewart R, Callard FJ, Moran P. Life expectancy at birth and all-cause mortality among people with personality disorder. J Psychosom Res 2012;73:104-7. http://dx.doi.org/10.1016/j.jpsychores.2012.05.001

13. Pompili M, Girardi P, Ruberto A, Tatarelli R. Suicide in borderline personality disorder: a meta-analysis. Nord J Psychiatry 2005;59:319-24. http://dx.doi.org/ 10.1080/08039480500320025 
14. Hörz S, Zanarini MC, Frankenburg FR, Reich DB, Fitzmaurice G. Ten-year use of mental health services by patients with borderline personality disorder and with other axis II disorders. Psychiatr Serv 2010;61:612-6. http://dx.doi.org/ 10.1176/ps.2010.61.6.612

15. Andrews G, Titov N. Changing the face of mental health care through needsbased planning. Aust Health Rev 2007;31 Suppl 1:S122-8. http://dx.doi.org/ 10.1071/AH07S122

16. National Health and Medical Research Council. Clinical Practice Guideline for the Management of Borderline Personality Disorder. Melbourne: National Health and Medical Research Council; 2012.

17. Leichsenring F, Leibing E, Kruse J, New AS, Leweke F. Borderline personality disorder. Lancet 2011;377:74-84. http://dx.doi.org/10.1016/S0140-6736(10)61422-5

18. American Psychiatric Association. Diagnostic and statistical manual of mental disorders. 5th ed. Washington (DC): American Psychiatric Association; 2013.

19. Zimmerman M, Chelminski I, Young D, Dalrymple K, Martinez J. Does the presence of one feature of borderline personality disorder have clinical significance? Implications for dimensional ratings of personality disorders. J Clin Psychiatry 2012;73:8-12. http://dx.doi.org/10.4088/JCP.10m06784

20. Tyrer P, Reed GM, Crawford MJ. Classification, assessment, prevalence, and effect of personality disorder. Lancet 2015;385:717-26. http://dx.doi.org/ 10.1016/S0140-6736(14)61995-4

21. Chanen AM. Borderline personality disorder in young people: are we there yet? J Clin Psychol 2015;71:778-91. http://dx.doi.org/10.1002/jclp.22205

22. Crawford TN, Cohen P, First MB, Skodol AE, Johnson JG, Kasen S. Comorbid Axis I and Axis II disorders in early adolescence: outcomes 20 years later. Arch Gen Psychiatry 2008;65:641-8. http://dx.doi.org/10.1001/archpsyc.65.6.641

23. Cohen P, Crawford TN, Johnson JG, Kasen S. The children in the community study of developmental course of personality disorder. J Pers Disord 2005:19:466-86. http://dx.doi.org/10.1521/pedi.2005.19.5.466
24. Stoffers JM, Völlm BA, Rücker G, Timmer A, Huband N, Lieb K. Psychological therapies for people with borderline personality disorder. Cochrane Database Syst Rev 2012;8:CD005652.

25. Bateman AW, Gunderson J, Mulder R. Treatment of personality disorder Lancet 2015;385:735-43. http://dx.doi.org/10.1016/S0140-6736(14)61394-5

26. De Panfilis C, Marchesi C, Cabrino C, Monici A, Politi V, Rossi M, et al. Patient factors predicting early dropout from psychiatric outpatient care for borderline personality disorder. Psychiatry Res 2012;200:422-9. http://dx.doi.org/10.1016/j.psychres.2012.03.016

27. Stoffers J, Völlm BA, Rücker G, Timmer A, Huband N, Lieb K. Pharmacological interventions for borderline personality disorder. Cochrane Database Syst Rev 2010;6:CD005653.

28. National Collaborating Centre for Mental Health. Borderline personality disorder: treatment and management. Report No.: CG78. London: National Institute for Health and Clinical Excellence; 2009.

29. Stoffers JM, Lieb K. Pharmacotherapy for borderline personality disorder-current evidence and recent trends. Curr Psychiatry Rep 2015;17:534. http://dx.doi.org/10.1007/s11920-014-0534-0

30. Lieb K, Völlm B, Rücker G, Timmer A, Stoffers JM. Pharmacotherapy for borderline personality disorder: Cochrane systematic review of randomised trials. Br J Psychiatry 2010;196:4-12. http://dx.doi.org/10.1192/bjp.bp.108.062984

31. Tyrer P, Silk KR. A comparison of UK and US guidelines for drug treatment in borderline personality disorder. Int Rev Psychiatry 2011;23:388-94. http://dx.doi.org/10.3109/09540261.2011.606540

32. Zanarini MC, Frankenburg FR, Hennen J, Silk KR. Mental health service utilization by borderline personality disorder patients and Axis II comparison subjects followed prospectively for 6 years. J Clin Psychiatry 2004;65:28-36. http://dx.doi.org/10.4088/JCP.v65n0105

\section{Book review}

\section{Handbook of drug administration via enteral feeding tubes. 3rd ed.}

\author{
White R, Bradnam V \\ London: Pharmaceutical Press; 2015. \\ 732 pages
}

This new edition covers over 400 drugs and includes 29 new monographs. It aims to support safe and effective prescribing by providing practical recommendations on the administration of drugs via enteral tubes. Information in the book comes from pharmaceutical companies, published information (which is limited), and research undertaken largely by pharmacists.

The introductory chapters cover technical and pharmaceutical issues with tubes, drugs and administration, as well as tube blockage, drug errors and occupational exposure.

The monographs list UK formulations and relevant data for each drug. Information is provided on sites of oral absorption, alternative routes, interactions with food and enteral feeds, health and safety precautions, altering available products, and intragastric and intrajejunal administration procedures.
The products listed may differ from those available in Australia. The print is very small, and the book does not cover administration to patients with swallowing difficulties. A publication by the Society of Hospital Pharmacists of Australia, the Australian Don't Rush to Crush Handbook, covers administration via enteral tubes in less detail, but includes information on swallowing difficulty and products available in Australia. There is some overlap between the two books, but each contains unique content.

This is a practical book which contains more detail on drug administration via enteral tubes than is readily available elsewhere. Because such administration is generally 'off-label' (outside the approved product information), those prescribing, dispensing or administering drugs via enteral tubes may be liable if the patient experiences adverse effects due to the route or alteration of the product. This makes reliable information on the topic particularly valuable. The handbook would be useful to nurses, pharmacists, doctors and dietitians who regularly deal with patients on enteral feeds.

\section{Leone Snowden}

Specialist pharmacist NSW Medicines Information Centre

Sydney

Aust Prescr 2016:39:53

http://dx.doi.org/10.18773/ austprescr.2016.004

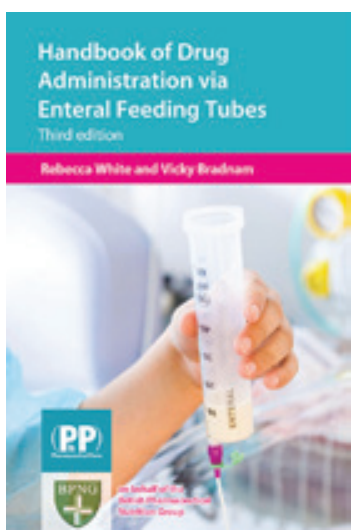

